\title{
What is supersymmetry?
}

Michael Atiyah

Supersymmetry and its Applications: Superstrings, Anomalies and Supergravity.

Edited by G.W. Gibbons, S.W. Hawking and P.K. Townsend.

Cambridge University Press: 1986. Pp.481. £27.50, \$49.50.

Physicists are an ambitious lot. Their aim is to understand the Universe and what makes it tick. What is matter? What are the forces that act on it? How did it start and where will it end? These, no less, are the monumental questions that theoretical physicists attempt to answer. So far, in historical terms, their track record in answering these questions is quite impressive. The two great triumphs of earlier centuries, Newton's theory of gravitation and Maxwell's theory of electromagnetism, remain outstanding examples of what a physical theory should be. Universal in application, and capable of simple and rigorous formulation in mathematical terms, they provide a firm basis from which to attack complex and realistic problems.

At the core of both theories are the notions of "particles" and "fields", that is of matter and force. Particles produce fields and fields act on particles in a perpetual duet. As physicists have delved further into the nature of this interaction, the theories that have emerged have become more and more complex, both conceptually and mathematically. Einstein altered our view of the separateness of space and time, and his theory of general relativity is much more sophisticated than Newton's, although equally rigorous in its mathematical formulation. Quantum mechanics involved an even greater revolution and its foundations are much less clear. "Elementary particles" have been emerging from the laboratories in everincreasing numbers, and theories to explain them have been just as numerous.

In all this excitement and turmoil two main problems have always stood out. What are the ultimate "elementary particles"? And how does one reconcile general relativity with quantum mechanics? The first of these is closely tied to experimental discoveries and alters with each new generation of accelerators, while the second is a purely intellectual or mathematical problem because gravitation and quantum mechanics operate at quite different scales.

Quantum field theory aims to provide a theory of elementary particles consistent with quantum mechanics and based on a sophisticated unification of the notions of particle and field. The details of the theory may vary depending on how many kinds of particle one postulates. In fact, it is extremely difficult to construct any mathemati- cally consistent quantum field theory. Many theories are plagued with "ultraviolet divergences" or "anomalies" and the mathematical study of these problems has become quite an industry.

Given all the difficulties, it is not hard to understand why, in the past couple of years, there has been such widespread enthusiasm amongst theoretical physicists for what are called "superstring" theories. These theories appear to offer the first realistic chance of a consistent account of nature which combines quantum field theory and general relativity. As the term suggests, these new theories are a combination of tw̧o different ideas, namely strings and supersymmetry, both of which have been investigated separately for some time.

In one form or another, symmetry has always played a prominent part in physics. Essentially, the requirement that a physical law be independent of the particular location or orientation in space or time means that the relevant theory has to admit the appropriate symmetries of space-time. Beyond this physicists have found that elementary particles tend to come in families, and the best explanation has again been couched in terms of symmetries, not of space-time but of some "internal" space.

Particles in quantum mechanics come in two different species, depending on their "spin". There are fermions, which have half-integral spin and obey the Pauli exclusion principle, and bosons which have integral spin and have symmetric wavefunctions. Physically they are fundamentally different but supersymmetry ignores this difference and puts them on an equal footing. Roughly speaking, a supersymmetric theory should allow the interchange of fermions and bosons. Despite its physical implausibility supersymmetry has many theoretical attractions, and the mere requirement that a theory be supersymmetric is so strong that it greatly limits the number of possible candidates. For these reasons it has been extensively studied.

String theory starts from the idea that the idealized picture of a point-particle should be replaced by that of a string. This also has been studied, off and on, for many years with varying degrees of enthusiasm and optimism. The subject really hit the headlines however when, in its supersymmetric form, it was discovered by John
Schwarz of Caltech and Michael Green of Queen Mary College that a consistent anomaly-free theory could be constructed. This would in principle give a unified theory of elementary particles, incorporating gravity and quantum mechanics. Since then, there has been intense activity developing the several variants of the superstring theory and examining their consequences.

In 1985 a workshop in Cambridge, UK, was essentially devoted to these latest developments, and this volume records the various contributions. Many of the leading experts were present, and their talks give an authoritative view of the situation. While the material is highly technical, a few talks were addressed to the uninitiated, notably the introduction to strings by Edward Witten of Princeton.

Although superstring theories hold great promise and have generated renewed optimism about unified field theories, they are still very mysterious. The foundations are not at all clear, and surprises are probably in store for us. At the mathematical level, strings are difficult to understand, but it is already clear that they will involve many new techniques and ideas. It should be emphasized that the unification of forces involved in superstring theory takes place way beyond the realms of direct experimental verification. Thus the main tests of the theory are those of internal mathematical consistency. However, these are extremely severe. In fact physicists would like to believe that there is only one possible consistent solution. All they have to do is find it!

Perhaps the final word should be given to Witten, one of the high-priests of the subject, who, having explained the peculiar mathematics of superstrings, goes on to say:

But the fact that these things work, that these seemingly bizarre rules give ways of computing things in quantum gravity, giving sensible results and finite answers and leading in many different directions to all kinds of beautiful areas in mathematics, is a very deep mystery probably one of the deepest which has ever been encountered in physics. It is unlikely that a proper understanding of this mystery will be found either soon or simply. But it will be worth the wait.

Sir Michael Atiyah is Royal Society Research Professor in the Mathematical Institute, University of Oxford, 24-29 St Giles, Oxford OXI $3 L B, U K$.

- Accounts of supersymmetry and associated topics are now reaching textbooks for advanced undergraduates and postgraduates. Four such books have appeared over the past few months and will be reviewed in a future issue of Nature: Introduction to Supersymmetry by P.G.O. Freund; Introduction to Supersymmetry and Supergravity by P. West; Supersymmetry, Superfields and Supergravity: An Introduction by P.P. Srivastava; and Supersymmetry: $A$ Decade of Development edited by P. West. 